\title{
A method of fabricating n-type thermoelements from galena concentrate
}

\author{
SUBHASIS BOSE \\ Energy Research Unit, Indian Association for the Cultivation of Science, Jadavpur, Calcutta \\ 700032, India \\ MS received 25 November 1993; revised 2 August 1994

\begin{abstract}
A process of fabricating n-type thermoelements by using abundantly available galena concentrate, including optimization of the sintering temperature and time, is reported in this paper. The results of variation of room temperature resistivity and thermoelectric power have been explained on the basis of inhomogeneities in the sample.
\end{abstract}

Keywords. Galena concentrate; thermoelements.

\section{Introduction}

In order to generate electrical power from waste heat sources, one needs to choose those materials which are available in abundance and are relatively cheap. Lead sulphide has been suggested as a possible choice, since it is available in the form of natural ore. Acharya et al (1986) fabricated a thermoelectric generator using n-type $\mathrm{PbS}$ elements with 'galena aggregate' $(\mathrm{PbS}>95 \%$ ), a type of ore available in certain pockets of lead ore mines, and p-type elements using galena concentrates obtained from beneficiation of galena-bearing rocks. It is the beneficiated ore of galena which is available in abundance. It is therefore considered worthwhile to study the possibility of using galena concentrate for fabricating $n$-type thermoelements. The elements made by pressing galena concentrate are p-type mainly because of large amounts of oxides of lead. The doping of such a material with $n$-type impurities therefore becomes difficult without removing the oxides. Further, the process of removal of oxides should be amenable to mass production techniques. Barclay and Bear (1971) suggested the use of sulphur for removal of oxides. However, availability of sulphur itself may be a bottleneck. Thus, it was thought proper to use a cheap and easily available material like charcoal for this purpose. In this paper we report the results of a study of (a) removal of oxides by a dry method using charcoal and a wet method involving washing with $\mathrm{MgCl}_{2}$ solution, and (b) doping of deoxidized materials followed by determination of optimum condition of sintering temperature and time to obtain higher thermoelectric power coupled with low resistivity.

\section{Experimental}

\subsection{Preparation of samples}

The galena concentrate used in this study was collected from Hindustan Zinc Limited, Zawar Mines, Rajasthan, India. A general chemical analysis of the material is presented in table 1. The material obtained was crushed to fine power in acetone (to avoid oxidation) and sieved in a set of sieves of various mesh sizes in a sieve-shaker. Pellets were made by cold-pressing the material of different sieve sizes. The room-temperature 
Table 1. General chemical analysis of galena concentrate collected from three different places in Zawar Mines, Rajasthan, India.

\begin{tabular}{|c|c|c|c|}
\hline Element/compound & $\begin{array}{l}\text { From Mochia } \\
\text { Mongra lodes }\end{array}$ & $\begin{array}{c}\text { From Magra } \\
\text { lodes }\end{array}$ & $\begin{array}{l}\text { From Zawar } \\
\text { Mala lodes }\end{array}$ \\
\hline $\mathrm{Pb}(\%)$ & $68 \cdot 2$ & $66 \cdot 5$ & $62 \cdot 2$ \\
\hline $\mathrm{Zn}(\%)$ & 5.7 & 1.9 & 45 \\
\hline $\mathrm{Fe}(\%)$ & $2 \cdot 1$ & 2.01 & 40 \\
\hline Insoluble $\%$ & $1 \cdot 1$ & $3 \cdot 5$ & 1.57 \\
\hline $\mathrm{Ca}$ (present in the & & & \\
\hline $\begin{array}{l}\left.\text { form of } \mathrm{CaCO}_{3}\right)(\%) \\
\mathrm{Mg} \text { (present in the }\end{array}$ & $2 \cdot 0$ & 1.65 & 0.88 \\
\hline form of $\left.\mathrm{MgCO}_{3}\right)(\%)$ & 0.65 & $1 \cdot 34$ & 0.48 \\
\hline Co(p.p.m.) & 8 & - & 24 \\
\hline Ni(p.p.m.) & 26 & $0.9 \%$ & 28 \\
\hline$M n(\%)$ & $0-04$ & $0-087$ & 0.076 \\
\hline $\mathrm{Cu}$ (p.p.m.) & 380 & 680 & 260 \\
\hline Cd(p.p.m.) & 320 & 50 & 238 \\
\hline $\mathbf{S}(\%)$ & $12 \cdot 9$ & $11 \cdot 70$ & $14 \cdot 3$ \\
\hline $\mathrm{Sb}$ (p.p.m.) & 560 & - & - \\
\hline$A g$ (p.p.m.) & 1020 & 130 & - \\
\hline $\mathrm{Al}_{2} \mathrm{O}_{3}(\%)$ & 1.77 & $1 \cdot 14$ & 0.24 \\
\hline
\end{tabular}

Table 2 Room-temperature resistivity and thermoelectric power of samples sieved at various mesh sizes.

\begin{tabular}{lccc}
\hline $\begin{array}{l}\text { Mesh sizes } \\
\text { (BSS) }\end{array}$ & $\begin{array}{c}\text { Mesh sizes } \\
(\mu \mathrm{m})\end{array}$ & $\begin{array}{c}\text { Room-temperature } \\
\text { resistivity } \\
(\text { ohm-cm) }\end{array}$ & $\begin{array}{c}\text { Room-temperature } \\
\text { thermoelectric } \\
\text { power }(\mu \mathrm{V} / \mathrm{K})\end{array}$ \\
\hline$-60+85$ & $-263+185$ & $84 \cdot 59 \mathrm{~K}$ & $\mathbf{-}^{*}$ \\
$-85+100$ & $-185+158$ & $75 \cdot 91 \mathrm{~K}$ & $\mathbf{- *}^{*}$ \\
$-100+120$ & $-158+131$ & $64 \cdot 15 \mathrm{~K}$ & 36 \\
$-120+150$ & $-131+105$ & $35 \cdot 77 \mathrm{~K}$ & 23 \\
$-150+200$ & $-105+79$ & $25 \cdot 15 \mathrm{~K}$ & $17 \cdot 5$ \\
$-200+250$ & $-79+61$ & $8 \cdot 83 \mathrm{~K}$ & $2 \cdot 00$ \\
$-250+300$ & $-61+53$ & $5 \cdot 210 \mathrm{~K}$ & $1 \cdot 85$ \\
-300 & -53 & $5 \cdot 208 \mathrm{~K}$ & \\
\hline
\end{tabular}

*Thermoelectric power could not be measured because of high resistivity of the sample.

resistivity and thermoelectric power of these samples are shown in table 2 . It is seen that all the samples show p-type conduction unlike the results of Bear (1977), where some fractions of ore having higher particle size $(>45 \mu \mathrm{m})$ indicated $n$-type conduction. Pressed samples using -250 mesh $(61 \mu \mathrm{m})$ exhibited the lowest resistivities and hence this fraction was used in further study.

The material was deoxidized by mixing it with $2 \% \mathrm{w} / \mathrm{w}$ activated charcoal, and then heated in nitrogen atmosphere at $770 \mathrm{~K}$ for $1 \mathrm{~h}$. In order to compare these results with those of Barclay and Bear (1971), some samples were also deoxidized using $2 \%$ w/w sulphur. The removal of oxides was checked by X-ray diffraction study. 
The deoxidized samples were then doped by mixing with $2 \mathrm{~mol} \%$ of lead chloride and $5 \mathrm{~mol} \%$ of lead and heated in nitrogen atmosphere at $770 \mathrm{~K}$ for $48 \mathrm{~h}$. The material was then cold-pressed to cylindrical pellets of $1.27 \times 10^{-2} \mathrm{~m}$ diameter and $1.92 \times 10^{-2} \mathrm{~m}$ length at a pressure of $77.6 \times 10^{6} \mathrm{~N} / \mathrm{m}^{2}\left(5 \mathrm{ton} / \mathrm{in}^{2}\right)$ applied for $60 \mathrm{sec}$ in a HS steel die. The pellets were then sintered in nitrogen atmosphere at different temperatures and for different times to determine optimum conditions to make n-type thermoelement.

Electrical resistivity and thermoelectric power of the samples were measured by applying aqua-dag contacts. The measuring set-up has been reported earlier (Chatterjee et al 1987a).

Differential thermal analysis (DTA) and thermogravimetric analysis (TGA) spectra of the samples were recorded using a Stanton Redcroft STA-780 thermal analyser at a heating rate of $10^{\circ} \mathrm{C} / \mathrm{min}$ in nitrogen atmosphere.

\section{Results}

\subsection{Deoxidation of the galena concentrate}

The X-ray diffractogram of the as-available samples and those treated with charcoal and sulphur were used to tabulate the $d$-values (table 3). It can be seen from this table that heating with carbon and sulphur results in elimination of lines corresponding to PbO, which shows removal of oxide. DTA and TGA spectra of a typical sample consisting of lead concentrate and carbon are depicted in figure 3. It shows that the sample starts losing weight at around $500 \mathrm{~K}$, and then a large change of weight is seen at around $725 \mathrm{~K}$. The DTA peak at $825 \mathrm{~K}$ seems to be associated with reaction of carbon with lead oxide. The peaks at $565 \mathrm{~K}$ and $725 \mathrm{~K}$ are likely to be associated with other impurities.

Table 3. X-ray diffraction studies of galena concentrate deoxidized with $2 \%$ charcoal and $2 \%$ sulphur.

\begin{tabular}{|c|c|c|c|c|}
\hline $\begin{array}{l}\text { Standard values of } \\
\text { lead sulphide and } \\
\text { lead oxide }(\mathrm{PbO})\end{array}$ & & $\begin{array}{c}\text { Fresh galena } \\
\text { concentrate } \\
\text { sample }\end{array}$ & $\begin{array}{c}\text { Galena concen- } \\
\text { trate heated } \\
\text { with } 2 \% \\
\text { charcoal }\end{array}$ & $\begin{array}{c}\text { Galena concen- } \\
\text { trate heated } \\
\text { with } 2 \% \text { sulphur }\end{array}$ \\
\hline $\begin{array}{l}\text { Corresponding } \\
\text { planes }(h k l)\end{array}$ & $d(\mathrm{~nm})$ & $d(\mathrm{~nm})$ & $d(\mathrm{~nm})$ & $d(\mathrm{~nm})$ \\
\hline \multicolumn{5}{|l|}{ For lead sulphide } \\
\hline 111 & 0.3429 & 0.3370 & $0 \cdot 3370$ & 0.3365 \\
\hline 200 & 0.2969 & 0.2900 & 0.2913 & 0.2910 \\
\hline 220 & 0.2099 & 0.2057 & 0.2057 & 0.2057 \\
\hline \multicolumn{5}{|l|}{ For $\mathrm{PbO}$} \\
\hline $\begin{array}{l}212 \\
022\end{array}$ & 0.1866 & 01886 & - & - \\
\hline
\end{tabular}

Standard values are obtained from Powder diffraction file, Card No. 5-0592(PbS) and $5-0570(\mathrm{PbO})$. 
3.2 Effect of sintering temperature on room-temperature resistivity and thermoelectric power of undoped galena concentrate samples

Figure 1 shows the variation of $\log$ room-temperature resistivity $(\log \rho)$ as a function of reciprocal of the temperature of sintering for three types of samples: (a) untreated beneficiated ore, (b) beneficiated ore treated with $2 \%$ sulphur, and (c) beneficiated ore treated with $2 \%$ carbon. In the case of untreated beneficiated ore the curve shows three activation energies $\left(E_{a_{1}}=0.41 \mathrm{eV}, E_{a_{2}}=0.022 \mathrm{eV}\right.$ and $\left.E_{a_{3}}=0.66 \mathrm{eV}\right)$. However, the curves for samples deoxidized with sulphur and carbon show only two activation energies (for sulphur, $E_{a_{1}}=0.054 \mathrm{eV}$ and $E_{a_{2}}=0.64 \mathrm{eV}$, and for carbon, $E_{a_{1}}=0.014 \mathrm{eV}$ and $\left.E_{a_{2}}=0.46 \mathrm{eV}\right)$.

Figure 2 shows the variation of room-temperature thermoelectric power as a function of sintering temperature. For all sintering temperatures the absolute value of thermoelectric power is always higher for the untreated beneficiated ore followed by the sample deoxidized with carbon and then the sample treated with sulphur as

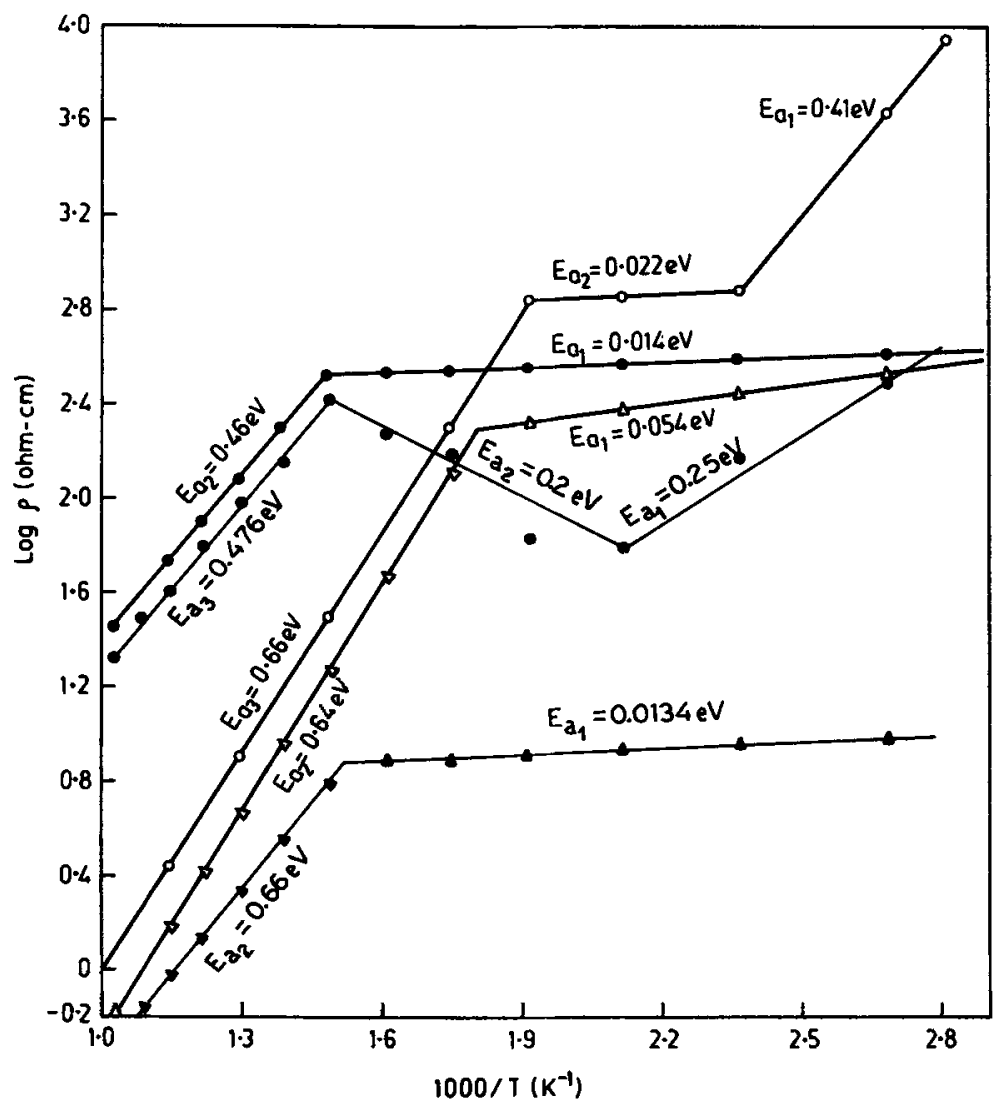

Figure 1. Room-temperature resistivity variation with the reciprocal of absolute sintering temperature of pellets made from galena concentrate $(O)$, galena concentrate treated with $2 \%$ sulphur $(\triangle)$, galena concentrate treated with $2 \%$ charcoal $(\Theta)$, galena concentrate sample washed with $\mathrm{MgCl}_{2}$ and doped with $\mathrm{PbCl}_{2}$ and $\mathrm{Pb}(\Theta)$, and galena concentrate deoxidized with charcoal and doped with $\mathrm{PbCl}_{2}$ and $\mathrm{Pb}(\Delta)_{3}$ for a fixed time of sintering $(1 \mathrm{~h})$. 
shown by figure 2 . Another feature of the curves for all the materials is the existence of a minimum in the value of thermoelectric power as the temperature of sintering is increased from room temperature to $973 \mathrm{~K}$. The temperature corresponding to the minimum depends on the treatment received by the sample.

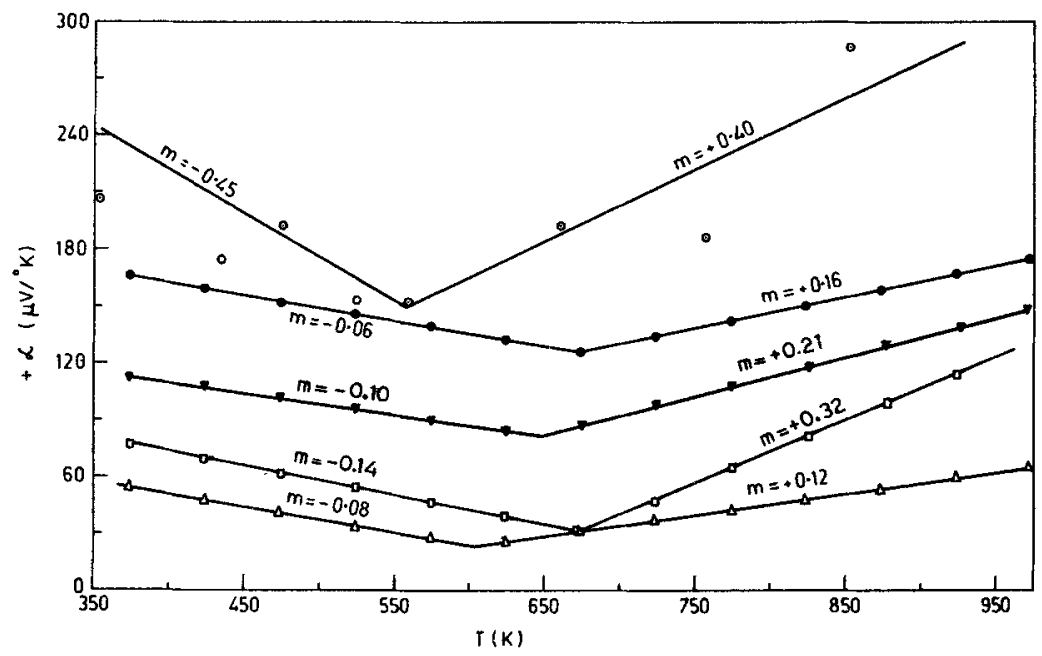

Figure 2. Room-temperature thermoelectric power variation with the absolute value of sintering temperature of pellets made from galena concentrate sample $(\odot)$, sample treated with $2 \%$ sulphur $(\triangle)$, sample treated with $2 \%$ charcoal $(O)$, sample washed with $\mathrm{MgCl}_{2}$ and doped with $\mathrm{PbCl}_{2}$ and $\mathrm{Pb}(\square)$, and sample deoxidized with charcoal and doped with $\mathrm{PbCl}_{2}$ and $\mathrm{Pb}(\boldsymbol{\nabla})$ for a fixed time of sintering $(1 \mathrm{~h})$.

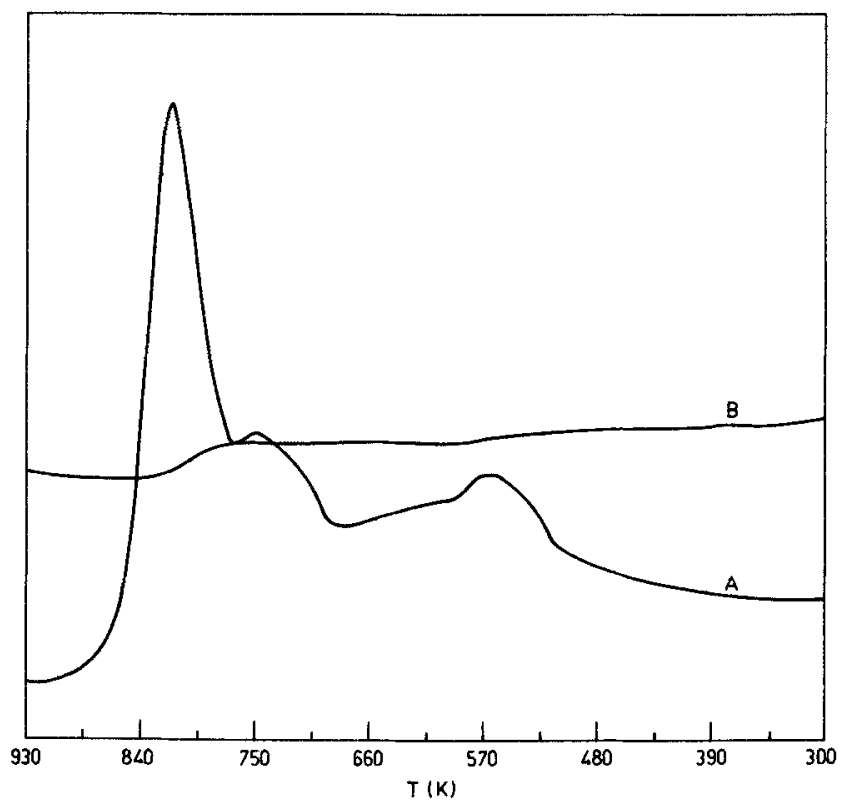

Figure 3. A. Differential thermal analysis (DTA) and B. thermogravimetric analysis (TGA) spectra for sample consisting of galena concentrate and charcoal. 
3.3 Effect of sintering temperature on room-temperature resistivity and roomtemperature thermoelectric power of deoxidized and doped samples of galena concentrate

Figure 1 shows the variation of $\log$ room-temperature resistivity $(\log \rho)$ as a function of reciprocal of the sintering temperature for samples (a) deoxidized with $0.25(\mathrm{~N})$ $\mathrm{MgCl}_{2}$ solution and doped with $5 \mathrm{~mol} \% \mathrm{~Pb}$ and $2 \mathrm{~mol} \%$ lead chloride, and (b) deoxidized by heating with $2 \%$ activated charcoal at $770 \mathrm{~K}$ in nitrogen atmosphere and doped as above. In the case of $\mathrm{MgCl}_{2}$-treated sample the curve shows three activation energies $\left(E_{a_{1}}=0.25 \mathrm{eV}, E_{a_{2}}=0.2 \mathrm{eV}, E_{a_{3}}=0.476 \mathrm{eV}\right)$, and the curve for the sample treated with activated charcoal shows two activation energies $\left(E_{a_{1}}=0.0134 \mathrm{eV}\right.$, $E_{a_{2}}=0.66 \mathrm{eV}$ ).

Figure 2 shows the variation of room-temperature thermoelectric power as a function of reciprocal of sintering temperature. The thermoelectric power decreases gradually with increment of sintering temperature up to a certain point and again starts increasing for both samples. However, the magnitude of the thermoelectric power for the samples treated with $\mathrm{MgCl}_{2}$ is always lesser than the sample treated with charcoal.

\section{Discussion}

All the samples made by cold-pressing of galena concentrate show p-type conduction irrespective of mesh size. This is in contrast with the results reported by Bear (1977), who got n-type conduction from pellets made with particle size less than $45 \mu \mathrm{m}$. This dissimilarity is likely to be associated with the chemical composition of grains. The lead content in Zawar Mines concentrate is lower compared to the concentrates used by Bear and Barclay (1977). Therefore, the Zawar Mines concentrate shows p-type conduction. The variation of room-temperature resistivity with the reciprocal of sintering temperature for the untreated beneficiated ore and the ore treated with charcoal or sulphur shows that the sintering takes place via surface diffusion in the low-temperature region and by volume diffusion in the high-temperature region similar to the results of Bear and Barclay (1975) and Chatterjee et al (1987b). The deoxidized material shows two clear-cut regions in contrast to the untreated ore, indicating that the process of deoxidation is complete. The doping of deoxidized material with lead and lead chloride results in n-type conduction and reduces the resistance as expected.

The variation of room-temperature resistivity with reciprocal of sintering temperature for the samples deoxidized by washing with $\mathrm{MgCl}_{2}$ solution followed by doping with $2 \mathrm{~mol} \%$ lead chloride and $5 \mathrm{~mol} \%$ lead shows a change of activation energy (slope) from low-temperature to high-temperature region. Such increased activation energies in the low-temperature region has been attributed to (Bear 1977) adsorption of impurities on the surface of the ore. These adsorbed substances desorb at a certain temperature and thus result in the change of the slope of the curve. Looking at the thermogravimetric spectrum of the sample heated with carbon it is seen that the sample starts losing weight at around $500 \mathrm{~K}$, which could be taken as evidence for desorption of adsorbed gases, and the absence of such a variation of activation energy for the samples deoxidized (clean) with carbon indicates that the treatment with carbon most likely removes all adsorbed gases and impurities from the surface of the grains. 
The p-type conduction in all the samples indicates that effectively the grains are still p-type in nature. The thermoelectric power of a semiconductor is given by

$$
\alpha_{p}=\frac{k}{e}\left[A+\ln \frac{N_{v}}{p}\right] .
$$

From (1) it is expected that as the resistivity decreases the thermoelectric power should also decrease. Looking at the resistivity curves for these samples at any temperature (figure 1), it is seen that

$$
\rho_{\text {beneficiated ore }}>\rho_{\text {carbon }}>\rho_{\text {sulphur }} .
$$

The thermoelectric power also shows the same expected behaviour. The minimum in the thermoelectric power curve has been explained by Chatterjee et al (1987b) on the basis of inhomogeneities in the sample enhanced by the process of sintering. Similar explanation should thus be possible in the present case. Figure 2 shows variation of the thermoelectric power with the reciprocal of sintering temperature for doped samples with a behaviour similar to undoped samples. If we look at the processing of the samples, one can imagine that the treatment with charcoal will result in deoxidation of the samples by removal of oxygen in the form of carbon dioxide and formation of lead atoms. The possible reaction is

$$
2 \mathrm{PbO}+\mathrm{C}=2 \mathrm{~Pb}+\mathrm{CO}_{2}
$$

The deposition of lead atoms on the surface of original p-type grain will result in the compensation of p-type impurities by these atoms. Further doping with lead chloride and lead will result in the conversion of outer layer of grains into n-type region, because both lead and chlorine are donors for the lead sulphide lattice. Such a sample

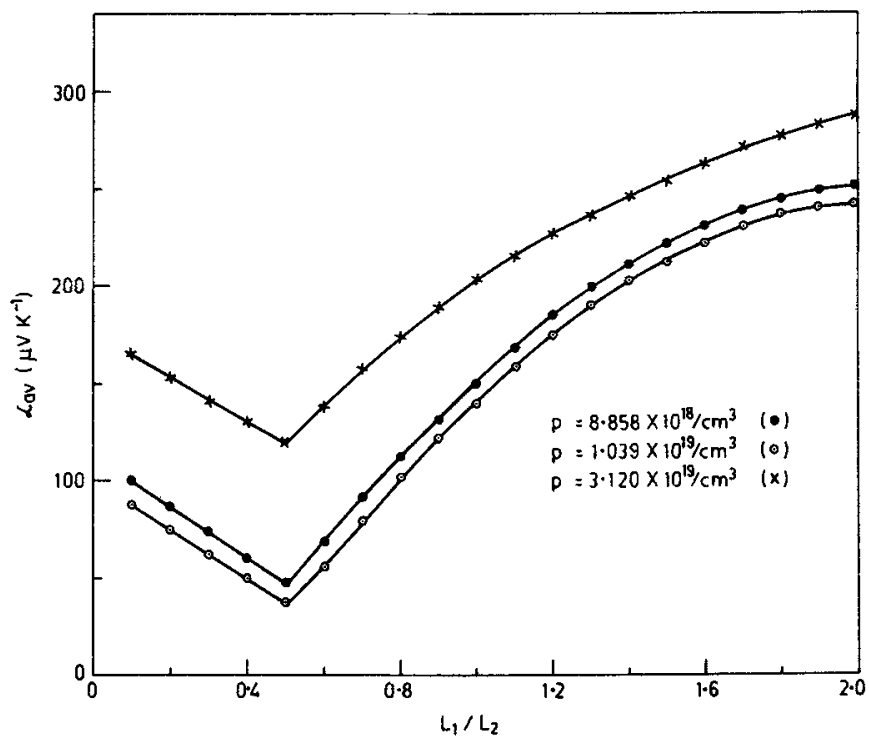

Figure 4. Variation of theoretically calculated thermoelectric power with $L_{1} / L_{2}$ for different values of $\mathrm{p}$ and fixed value of $\mathrm{n}=2.7 \times 10^{17} / \mathrm{cm}^{3}$. 
can then be thought of as consisting of grain whose inner core is of p-type of length $L_{2}$ and outer core is n-type of length $L_{1}$. The average thermoelectric power for such a system (Kwok 1980; Chatterjee et al 1987b) could be written as

$$
\alpha_{\mathrm{av}}=\frac{k}{e}\left[A\left(\frac{L_{1}-L_{2}}{L_{1}+L_{2}}\right)-\frac{L_{1}}{L_{1}+L_{2}} \ln \frac{N_{\mathrm{c}}}{n}+\frac{L_{2}}{L_{1}+L_{2}} \ln \frac{N_{\mathrm{v}}}{p}\right] .
$$

where $N_{\mathrm{v}}$ and $N_{\mathrm{c}}$ are effective density of states in valency and conduction band and $A$ a constant whose value is taken as 4 for $\mathrm{PbS}$ (Bloem 1956).

The process of sintering is therefore expected to change the relative magnitude of $L_{1}$ and $L_{2}$. At higher temperatures for the same time of sintering it is expected that the ratio $L_{1} / L_{2}$ will increase. A numerical computation of the value of $\alpha_{a v}$ as a function of $L_{1}$ and $L_{2}$ for certain assumed $\mathrm{n}$ and p-type carrier concentrations is shown in figure 4 . The figure shows that the thermoelectric power decreases with increase in the value of $L_{1} / L_{2}$ up to a value of approximately 0.5 . After this the thermoelectric power increases with increasing value of $L_{1} / L_{2}$. The qualitative variation of thermoelectric power with increasing sintering temperature can thus be understood in terms of change in the value of $L_{1} / L_{2}$ due to the heating process.

\section{Conclusions}

The study shows that it is possible to prepare n-type thermoelements of reasonably low resistivity $(\sim 0.30 \mathrm{ohm}-\mathrm{cm})$ and high thermoelectric power $(\sim 150 \mu \mathrm{V} / \mathrm{K})$ using abundantly available galena concentrate by dry deoxidation using charcoal followed by doping with lead and lead chloride. The optimum temperature of sintering is around $970 \mathrm{~K}$ and the time of sintering is $1 \mathrm{~h}$. The variation of the electrical properties of pellets with the temperature of sintering have been explained on the basis of inhomogeneities in the sample.

\section{References}

Acharya H N, Chatterjee S and Ratnam V V 1986 in Proceedings of the 6th int. conf. on thermoelectric energy conversion, Arlington, Taxas (Arlington: University of Taxas) p. 59

Barclay D V and Bear I J 1971 Solid State Commun. 91567

Bear I J 1977 Powder Met. Int. 972

Bear I J and Barclay D V 1975 Powder Met. Int. 7121

Bloem J 1957 Philips Res. Rep. 11173

Chatterjee S, Acharya H N and Ratnam V V 1987a J. Inst. Soc. India 1787

Chatterjee S, Acharya H N and Ratnam V V 1987b J. Mater. Sci. 222793

Kwok H L 1980 J. Phys. D13 441 